Chirurg 2019 $\cdot 90: 504$

https://doi.org/10.1007/s00104-019-0975-4

Online publiziert: 14. Mai 2019

(c) Springer Medizin Verlag GmbH, ein Teil von Springer Nature 2019

\section{Originalpublikation}

Nimptsch U, Haist T, Gockel I et al (2019) Complex gastric surgery in Germany-is centralization beneficial? Observational study using national hospital discharge data. Langenbeck's Arch Surg 404:93-101

Hintergrund und Fragestellung. Im Gegensatz zur Ösophaguschirurgie ist für die komplexe Magenchirurgie der Zusammenhang zwischen Fallzahl des Krankenhauses auf der einen Seite und den postoperativen Kurz- bzw. Langzeitergebnissen auf der anderen Seite in der Literatur nicht eindeutig geklärt, sodass gegenwärtig in Deutschland keine gesetzlich vorgeschriebenen Mindestmengen für diese Eingriffe existieren. $\mathrm{Zu}$ diesem Thema wurde eine aktuelle Analyse publiziert, welche ein realistisches Abbild der Magenchirurgie in Deutschland zeichnet und gleichzeitig die Frage der Zentralisierung zur Verbesserung des postoperativen Outcomes untersucht.

Methoden. Von 2010 bis 2015 wurden basierend auf den DRG-Datensätzen („diagnosis related groups“) mit den entsprechenden Diagnosen/Prozeduren nach ICD-10 und OPS (Operationenund Prozedurenschlüssel) alle stationären Patienten mit Magenresektion eingeschlossen. Primärer Endpunkt der Analyse war die Krankenhausmortalität in Abhängigkeit von der Fallzahl des Krankenhauses, als sekundärer Endpunkt wurde die Mortalität nach „Major“-Komplikationen („failure to rescue“) gewählt. Die Patienten wurden entsprechend dem Volumen des Krankenhaus in Quintilen unterteilt („very low“, „low“, „medium“, „high“, „very high“).

W. Schröder · C. J. Bruns

Klinik für Allgemein-, Viszeral- und Tumorchirurgie, Universitätsklinik Köln, Köln, Deutschland

\title{
Zentralisierung der komplexen Magenchirurgie in Deutschland
}

Ergebnisse. Insgesamt wurden in dem Untersuchungszeitraum die Datensätze von 72.528 stationären Patienten ausgewertet. Die Anzahl der jährlich durchgeführten Operationen sank signifikant von 12.773 in 2010 auf 11.281 in 2015, ebenso wie die Anzahl der operierenden Krankenhäuser (2010: 996; 2015: 892). Maligne Magentumoren waren mit $65 \%$ die häufigste Indikation zur Magenresektion, gefolgt von den Ulkuserkrankungen mit 14,6\%. Der häufigste Eingriff war mit $55 \%$ die subtotale (partielle) Magenresektion, (totale) Gastrektomien wurden bei $35 \%$ der Patienten durchgeführt. Die risikoadjustierte Krankenhausmortalität für das Gesamtkollektiv war in „Verylow-volume“-Krankenhäusern (Median 5 Eingriffe/Jahr, 12,0\%) signifikant höher als in „Very-high-volume“-Krankenhäusern (Median >50 Eingriffe/Jahr, $10,6 \%)$. Weiterhin wiesen „Very-highvolume“-Zentren eine signifikant geringere Krankenhausmortalität beim Vorliegen postoperativer Komplikationen auf (22,7\% vs. $28,1 \%)$. Signifikante Unterschiede in der Mortalität wurden insbesondere für die verschiedenen Indikationen zur Magenresektion beobachtet (Karzinomchirurgie $<8 \%$, Ulkuschirurgie $>25 \%$ ). Bei 47.436 Magenresektionen wegen eines primären Karzinoms war die Krankenhausmortalität in den „Very-high“- gegenüber den „Very-lowvolume“-Krankenhäusern signifikant geringer (6,3\% vs. $7,7 \%)$.

Fazit. Die beobachteten Mortalitätsraten sind insbesondere von der Indikation zur Magenresektion abhängig, jeder vierte Patient verstirbt nach der Resektion eines gastralen Ulkus. Die unterschiedlichen Krankenhausmortalitäten in den verschiedenen Versorgungsstufen sind am ehesten durch ein effektiveres Komplikationsmanagement der spezialisierten Zentren zu begründen. Auffällig ist aber, dass die Mortalitätsunterschiede lediglich signifikant zwischen den „Verylow“- und „Very-high-volumen“-Krankenhäusern sind, nicht aber für die Stufen dazwischen („low“, „medium“ und „high“). Daher folgern die Autoren, dass nur eine Zentralisierung mit chirurgischen Abteilungen, die mehr als 50 Eingriffe pro Jahr durchführen, die Krankenhausmortalität positiv beeinflussen würde.

\section{Korrespondenzadresse}

Prof. Dr. W. Schröder, FACS, FEBS

Klinik für Allgemein-, Viszeral- und Tumorchirurgie, Universitätsklinik Köln Kerpener Str. 62, 50937 Köln, Deutschland wolfgang.schroeder@uni-koeln.de

Interessenkonflikt. W. Schröder und C.J. Bruns geben an, dass kein Interessenkonflikt besteht. 